\title{
Clinical challenges posed by new biotechnology
}

\section{Castle}

\section{The case of nutrigenomics}

f all goes as planned, the world in which we can obtain an inexpensive, easy to produce digital data version of our personal genome is not far in the future. ${ }^{1}$ The idea of possessing genetic self knowledge is intriguing. Imagine holding an optical storage disk encoded with your genome, a disk whose contents can reveal a staggering amount about your genetic identity, past, present, and future. Having genetic self knowledge is also empowering. The disk containing your personalised genomic knowledge could enable you to make informed choices and take life transforming steps to prevent or mitigate disease.

If complete genome DVDs sound futuristic, it is because data collection and encoding issues stand in the way. Technical problems will undoubtedly be finessed. When they are, we will realise that beneath the initial allure of improved individual access to genetic information lies a more interesting phenomenon. We are witnessing the rapid movement of genomic science and technology into the commercial sector fuelled by the progress being made in the elucidation of the identity, function, variants, and interaction of our 30000 genes. Perhaps the most significant outcome of the published draft sequences ${ }^{23}$ of the human genome was the nearly fourfold reduction in the predicted number of human genes. Once one gene-one effect determinism was decisively overthrown, pleiotropy, polygenic effects, and gene-environment complexities suddenly became the orthodoxy in biology. Genomics, once ridiculed by Syndey Brenner as work so boring that inmates should do it, has produced very complex results. Uptake of genomics into medical genetics likewise reflects the subtlety and variety of the science under girding predictions about disease susceptibility, ${ }^{45}$ behavioural dispositions, ${ }^{6}$ and heterogeneous response to drugs. ${ }^{7}$ Intensive diversification is the hallmark of genomics science and technology, and tremendous opportunity exists for entrepreneurial conversion of human genomics into viable commercial ventures. For the foreseeable future, expect the proliferation of business development in human genomics.
On the frontier of commercialised genetic technologies is nutrigenomics. Nutrigenomics is shorthand for nutritional genomics, which, as the name implies, is the study of the interaction between nutrients and genes. Human genomics and associated biotechnologies arising from the Human Genome Project are being conjoined with nutritional science to study heterogeneous responses to nutrient intake. While much of this research is based on population level studies, nutrigenomics also encompasses the complex interaction between nutrient sensitivity and disease through individual variation. ${ }^{8}$ The latter involves personalised genetic testing, the results of which are fitted to a diet individually tailored for disease prevention, mitigation, or modulation. Specific nutrients are made available through conventional foods, functional foods and nutraceuticals, and it is possible to genetically modify foods to enhance or introduce derived nutrient output traits.

Nutrigenomics is at work, for example, in the identification of polymorphisms in the gene for the methylene tetrahydrofolate reductase (MTHFR) enzyme. MTHFR regulates folate and is active in maintaining acceptable homocysteine levels. A common mutation, C677T, decreases MTHFR activity, increasing the incidence of homocysteinuria-a cardiovascular disease risk factor. ${ }^{90}$ Once this polymorphism is identified, folate supplemented diets can lower homocysteine levels, thereby preventing potential cardiovascular disease. Genetic tests for polymorphisms in the MTHFR gene are inexpensive and easy to administer, and have the potential to reap significant health benefits.

Nutrigenomics is driven into the marketplace by a combination of business savvy and access to current human genomic science and technology. Nutrigenomics is drawn into the marketplace by growing consumer interest in having direct control over nutrition and health. Consumer research conducted by groups like the California based Institute for the Future strongly indicate that over the next decade, consumers will continue to diversify the range and kind of nutritional and health information sources available to them. ${ }^{11}$ As they become more knowledgeable and adventurous, a larger market for personalised health and dietary information will flourish. It is already recognised that the emphasis in Western society on consumer choice and individual autonomy will drive health care in the direction of private testing. ${ }^{12}$ The resulting trend is for consumers to take more initiative and seek out their own nutritional advice. Consumers will combine nutritional advice gained from several sources, but the trend is for them to seek information, even counselling in some cases, on the web. ${ }^{13}$ New consumers are making independent dietary decisions, and their actions will have a knock-on effect on the growth of the nutrigenomics marketplace.

Ignorance shrouds any assessment of the clinical impact of nutrigenomics. The technology is too new to have been studied systematically, and just one company has provided nutrigenomics to consumers. ${ }^{14}$ Nevertheless, it is patently obvious that some of the clinical issues that are relevant to genomics based medicine will dog nutrigenomics too. For example, incipient technologies always produce new distributions of benefits and potential harms to patients. Another set of perennial concerns arise anytime genetic material is collected and analysed. Worries about patient disclosure protocols, the protection of privacy, and the threat of genetic discrimination prevail.

In addition to these considerations, specific clinical challenges associated with nutrigenomics are foreseeable. Under a patient model, individuals are tested and advised by physicians within a clinical context. Patients seeking nutrigenomics services in this way will put new demands on physicians to provide genetic testing and test evaluations. Resource shortfalls will preclude universal distribution of nutrigenomics tests, thereby generating a patient group further stratified by access inequities. Suppose, however, that nutrigenomics tests become widely available. In this case a different problem arises as greater numbers of physicians will find themselves in unfamiliar territory. Nutrigenomics offers presymptomatic tests, the results of which may not lead to disease diagnoses but to estimates of one's propensity for a variety of diseases based on a set of polymorphisms. Clinical practitioners normally do not order such broad tests, tests not prompted by symptoms, nor do they routinely order prophylactic tests. Instead, patient testing is usually narrowly focused, based on indicators of extant disease, and tests are ordered with the hopes of alleviating symptoms and curing underlying causes. Nutrigenomics is more preventative than curative, and only becomes preventative once a person's risks factors have been identified. 
For the physician prescribing tests in response to patient demand for nutrigenomics, there is no way to distinguish between the sick and the worried well. ${ }^{15}$ It is unclear how physicians should to respond to patient demands for nutrigenomics when many basic health needs are not being met in over-taxed health care systems. Nutrigenomics may lie beyond the realistic grasp of preventative medicine.

In a consumer model, nutrigenomics testing and nutritional consultation is offered direct to the consumer. Physicians are subsequently consulted regarding the results of the genetic test and diet recommendations. Consumers who seek services outside of the clinical context will perhaps pose the greatest challenge to physicians. Suppose a consumer of a direct-to-consumer nutrigenomics test finds they have mutation for which dietary intervention is recommended, but they require further consultation with a physician. The consumer will then switch modes to being a patient, but the burden of correctly interpreting nutrigenomics advice generated outside of the clinical context suddenly falls on the physician. Without the consultation, a patient may make inappropriate decisions with potentially grave repercussions. Conversely, successful consultation depends upon physicians' ability to properly interpret genetic tests they did not administer, and about which they may know little or nothing. If it is unreasonable to ask physicians to double as proficient genetics counsellors, and to ask them to gain detailed knowledge in the more narrowly focused field of nutrigenomics, will patients learn to trust sources other than their doctors? As it is, early adopters of nutrigenomics tend have antecedent health and nutritional knowledge collected from sources other than their physician. They may be predisposed to trust physicians less.

It remains the case that nutrigenomics poses serious questions about the role of nutrition in clinical medicine. Is nutrigenomics fundamentally nutritional counselling buttressed by genetic testing, or is it medical genetics counselling with dietary recommendations in place of pharmaceutical prescriptions? Physicians and their professional associations will have to decide whether nutrigenomics falls under medicine's jurisdiction or under the watch of nutritionists, and patients and regulators will need to know who is accountable for nutrigenomics health claims and the provision of nutrigenomics services. The seriousness of this problem is obvious if one considers that like pharmacogenomics and other genomics technologies, nutrigenomics will do its part to further perpetuate the belief that genetic tests definitively predict future health. ${ }^{16}$ Unethical promotion of nutrigenomics based on hyperbolic statements about health benefits may lead to false expectations of deterministic outcomes when probabilistic statements are appropriate. Unlike pharmacogenomics, which falls within a pre-existing and well developed pharmaceutical regulatory regimen that provides physicians with a measure of control over the information flow, nutrigenomics may lapse into the functional food and nutraceutical regulatory quagmire. The longer nutrigenomics resides in regulatory limbo between medicine and nutrition, physicians are more likely to be on the front lines educating the public about the nature of nutrigenomic risk factors and their management and dispelling myths about genetic determinism. Physicians will face this burden regardless of whether people's first contact with nutrigenomics follows a consumer or patient model. These issues are inevitable because we are much closer to having widespread access to personal genome DVDs than we are to knowing how to manage properly the context in which genomics information will be used.

Postgrad Med J 2003;79:65-66
Author's affiliation

D Castle, Department of Philosophy, University of Guelph, Guelph, Ontario, Canada NIG $2 \mathrm{Wl}$

Correspondence to: Dr Castle;

dcastle@uoguelph.ca

\section{REFERENCES}

1 Pollan M. Genome pioneer will start center of his own. New York Times 15 August 2002: 18 (A).

2 Venter C, Adams MD, Meyers EW, et al. The sequence of the human genome. Science 2001;291:1304-51.

3 International Human Genome Sequencing Consortium. Initial sequencing and analysis of the human genome. Nature 2001;409;860-921.

4 Hall JM, Lee MK, Newman B, et al. Linkage of early-onset familial breast cancer to chromosome 17q21. Science 1990;250: 1684-9.

5 Venkitaraman A. Functions of BRCA1 and BRCA2 in the biological response to DNA damage. J Cell Sci 2001;114:3591-8.

6 Caspi A, McClay J, Moffitt TE, et al. Role of genotype in the cycle of violence in maltreated children. Science 2002;297:851-4.

7 Nebert DW, Bingham E. Pharmacogenomics: out of the lab and into the community. TRENDS in Biotechnology 2001;19:519-23.

8 Elliott R, Ong J. Nutritional genomics. BM 2002; 324: 1438-42.

9 Frosst P, Blom HJ, Milos R, et al. A candidate genetic risk factor for vascular disease: a common mutation in methylenetetrahydrofolate reductase. Nat Genet 1995;10:111-3.

10 Verhoef $\mathbf{P}$, Rimm EB, Hunter DJ, et al. A common mutation in the methylenetetrahydrofolate reductase gene and risk of coronary artery disease: results among US men. J Am Coll Cardiol 1998;32:353-9.

11 Institute for the Future. The future of nutrition: consumers engage with science. Menlo Park, CA: Institute for the Future, 2001

12 Caulfield T, Burgess MM, Williams-Jones $B$, et al. Providing genetic testing through the private sector: a view from Canada. Isuma 2001 ;Autumn:72-81.

13 Guttmacher AE. Human genetics on the web. Annual Review of Genomics. Human Genetics 2001;2:213-33.

14 Sciona. Available on: www.sciona.com (accessed 4 September 2002).

15 Human Genetics Commission. The supply of genetic tests direct to the public: a consultation document. London: Human Genetics Commission, 2002.

16 Pharmacogenomics Consortium. Pharmacogenomics: ethical and regulatory issues in research and clinical practice. University of Minnesota: University of Minnesota Centre for Bioethics, 2002. 